\title{
COMMENTARY
}

\section{The Imperative to Expand Provision, Access and Use of Misoprostol for Post-Abortion Care in sub-Saharan Africa}

\author{
Andrzej Kulczycki
}

Associate Professor, Department of Health Care Organization \& Policy, University of Alabama at Birmingham (UAB), 320 Ryals Public Health Bldg., 1665 University Blvd., Birmingham, AL 35294-0022, USA

*For Correspondence: E-mail: andrzej@uab.edu

The number of maternal deaths due to unsafe abortions has fallen worldwide since the early $1990 \mathrm{~s}^{1}$. However, the situation in sub-Saharan Africa (SSA) remains a source of great concern for multiple reasons: abortion rates have not fallen, clandestine abortions are common and often deadly, and post-abortion care (PAC) for women experiencing problems from spontaneous or induced abortion remains deficient. Abortion remains largely outlawed nearly everywhere ${ }^{2}$, which pushes the practice into a dangerous and shadowy underground market. This confluence of factors leads to high rates of preventable abortionrelated morbidity and mortality.

Additionally, both domestic and transnational groups have lobbied against the expansion of legal indications for abortion and even against access to family planning services ${ }^{3}$, which could help reduce unintended pregnancies and the frequent resort to abortion. Contraceptive uptake lags behind other world regions and unmet need is high, double the world average; in 28 SSA countries, including all but one in West Africa, fewer than $25 \%$ of women of childbearing age used contraception, with unmet need as high as $36 \%{ }^{4}$. In many African countries, contraceptive prevalence rates have stagnated and family planning efforts are weak. To reduce the public health burden, policies are needed to help reduce the incidence and consequences of abortion, especially unsafe abortion.

Globally, the ratio of abortion-related deaths per 100,000 live births has declined due to improvements in abortion-related technology, clandestine use of abortion-inducing drugs and, to a lesser extent, increases in improved PAC. These developments are most apparent in Latin America whereas in SSA, abortion remains largely outlawed and abortion rates are similar ${ }^{5}$, but the ratio of abortion-related deaths to live births was estimated at 10/100,000 in 2008, as opposed to 80 in Africa ${ }^{1}$. In Africa's largest country, despite legal restrictions, approximately 1.25 million Nigerian women were estimated to have had an abortion in 2012 and $40 \%$ of them experienced complications serious enough to require treatment ${ }^{6}$. Abortion-related mortality (and morbidity) is becoming a particularly African problem.

PAC is an effective intervention, involving emergency treatment of abortion-related complications and post-abortion family planning $^{7,8}$. It is also an ethical and humanitarian imperative. However, SSA endures major unmet needs for accessible, quality, and sustainable PAC services, even in countries with permissive abortion laws such as Ghana and Zambia. Even in South Africa, which has legalized abortion on broad grounds, PAC services are often substandard. Poor and rural women in particular suffer the consequences. Across SSA, innovative, low-cost solutions are needed to manage complications from unsafe abortion, particularly in rural and remote areas.

\section{Misoprostol use in SSA}

In many African countries, misoprostol has recently become well-known in hospital settings for various obstetric uses and is included in the Ministry of Health's essential drugs list ${ }^{10}$. Misoprostol was originally used to treat ulcers and is increasingly viewed as an important medication for postpartum hemorrhage. Its safety, effectiveness, appropriate regimens, and acceptability are now well established for such purpose. Misoprostol is heat-stable, relatively inexpensive, and easy to administer. It is now also being seen as a potentially viable option for 


\section{Andrzej Kulczycki}

treating incomplete abortion and miscarriage ${ }^{11-13}$. In 2009, Nigeria became the first country in the world to add misoprostol to its essential medicines list for PAC. Other African countries have since followed, yet many barriers exist to effective deployment of misoprostol for PAC (MPAC). Misoprostol can be used to induce first trimester abortions and resistance to making such procedures legal may inadvertently block access to the life-saving potential of MPAC. Misoprostol is typically not obtained through regular health service channels and may be associated with substandard care and used incorrectly. Furthermore, there are many gaps in the literature on service delivery aspects of misoprostol, particularly for MPAC, hindering efforts to institutionalize its more widespread safe and effective use.

The Millennium Development Goals are now replaced by 17 Sustainable Development Goals (SDGs) and a new phase of extending international development efforts is about to begin. One way to help realize the goal to improve health and reduce inequities would be to help ensure MPAC. Incomplete abortion can cause lifethreatening complications without well-timed and proper treatment, including effective contraceptive method provision and counseling. Recent studies indicate no significant differences in effectiveness and safety between treatment of unsafe abortion with misoprostol and surgical intervention. Thus, MPAC is an effective, low-cost alternative to surgical techniques that could be applied in lowresource settings, including rural areas with severely restricted access to surgical care.

Misoprostol should be readily available for women who do not otherwise have access to PAC. MPAC is a pragmatic response to physician shortages and there is need to build additional evidence supporting task-shifting in PAC and development of an effective approach to MPAC provision across health system levels. We know that community-based birth attendants and women themselves can be trained to use misoprostol to prevent post-partum hemorrhage. Furthermore, mid-level providers (MLPs) can safely provide first-line PAC services, including MPAC in outpatient settings, if given appropriate training and sufficient support. A recent Ugandan study showed midwives can offer MPAC equally

\section{Misoprostol for post-Abortion Care in Africa}

effectively and safely as physicians ${ }^{14}$. However, findings centered about Kampala, the capital city. Misoprostol's characteristics make it a smart option for treating incomplete abortion in SSA, especially at the district and sub-hospital levels in remote, rural and impoverished areas where access to PAC is most restricted, but this has yet to be demonstrated and effectively operationalized at mid- and lower-level facilities.

\section{The way forward}

The next steps to improve the situation in Africa need to be taken in public health systems and family planning, which is essential to preventing unintended pregnancies and abortions. The problem is not just one of lack of resources and capacity for implementation; action is pre-empted by a mix of fears about confronting cultural stigma, turf issues, and inertia, leading to risk aversion. This situation requires working with and empowering MLPs, educating the full range of providers, informing women and communities, and incorporating MPAC into routine reproductive and primary health care.

These actions could be supplemented by encouraging or requiring open-ended, judgementfree informational counseling for women and communities. This is important given the limited control that most African women have over their reproductive lives and poor reproductive health knowledge. The counselling could also provide information on how MPAC addresses public health concerns, and embolden providers to speak out about the costs involved and the means to take action immediately to treat complications from unsafely performed abortions. This would help alter public perceptions about the benefits of misoprostol.

Achieving further progress requires continued advocacy for reproductive and sexual rights, as well as sound public health programming. This should include better adherence to international conventions such as the Maputo Protocol to the African Charter on women's rights and the associated Plan of Action on Sexual and Reproductive Health and Rights, which both came into force in the last decade ${ }^{15}$. There is need for an expansive view of sexual and reproductive health rights, which is essential also 
Andrzej Kulczycki

to reducing glaring health inequalities. Reproductive rights are rooted in basic human rights and are important to health, particularly for women, but they remain subject to powerful social, political, and other determinants.

More research is needed to help build the weak evidence base on how best to operationalize MPAC, particularly in rural and poor communities where access is limited, health care providers are fewer, unmet reproductive health care needs are greatest, and risks of unsafe abortion are highest. This would strengthen efforts to improve maternal health through task-shifting, especially to midwives, whose ability to administer MPAC may help countries with major health workforce shortages and high levels of pregnancy-related mortality and morbidity.

\section{Conclusion}

SSA has high levels of unintended pregnancy, low rates of modern contraceptive use, and weak health system capacity. Despite legal restrictions and its controversial nature, abortion is more common in much of SSA than is acknowledged or often realized, and because it tends to be clandestine and unsafe, it is frequently dangerous and costly to individuals, their families, and health systems. This situation contributes substantially to maternal mortality. Consequently, PAC is an ethical, humanitarian and public health obligation for reducing maternal mortality and morbidity.

There is a strategic logic to an incremental approach to this issue, one on which many people and advocates on all sides have ideal points far from the status quo, and legislators often hold incomplete information, as well as their own particular agendas. PAC is often connected in the minds of politicians and in the public consciousness with the legal status of abortion, an issue that sparks much passion and is often insinuated into politics centered in arguments about gender, religion, and even national identity. However, improvements in access to contraceptive services and in the provision of quality MPAC services (as permitted by law, international treaties and conventions) may help reduce maternal morbidity and mortality.

MPAC is a promising intervention that can

\section{Misoprostol for post-Abortion Care in Africa}

mitigate physician shortages, save resources, and reduce costs of unsafe abortion. It is particularly pertinent to district and sub-hospital levels in remote, rural and impoverished areas, where access to PAC and surgical care are most restricted. The addition of MPAC in outpatient settings to the regimen of care by MLPs who would also provide post-abortion family planning could help to significantly reduce maternal illhealth.

An expansive view of sexual and reproductive health rights with open-ended informational counseling are needed to help better provide MPAC. Studies are needed to help operationalize the delivery of quality misoprostol care as a primary treatment for incomplete abortion in lower-level facilities, especially at the sub-hospital level, and to scale-up effective approaches. The time to act is now, given insufficient attention paid to this effective, lowcost intervention to date and that the UN's new SDGs have yet to come into force.

Conflict of Interest: None

\section{References}

1. WHO. Unsafe Abortion: Global and Regional Estimates of the Incidence of Unsafe Abortion and Associated Mortality in 2008. Geneva: World Health Organization, $6^{\text {th }}$ ed., 2011.

2. UN. Abortion policies \& reproductive health around the world. New York: United Nations, 2014.

3. Kulczycki A. The Abortion Debate in the World Arena. London: Macmillan and New York: Routledge, 1999.

4. UN. Trends in Contraceptive Use Worldwide 2015. New York: United Nations, 2015.

5. Kulczycki, A. Abortion in Latin America: Changes in Practice, Growing Conflict, and Recent Policy Development. Studies in Family Planning, 2011; 24(3): 199-220.

6. Bankole A, Adewole IF, Hussain R, Awolude O, Singh $\mathrm{S}$, Akinyemi J0. The incidence of abortion in Nigeria. International Perspectives on Sexual and Reproductive Health, 2015; 41(4):170-181.

7. USAID, FIGO, Population Council FRONTIERS, Health Communication Partnership (2007) What works: A policy and program guide to the evidence on postabortion care. Washington, DC: USAID, FIGO, Population Council FRONTIERS, Health Communication Partnership.

8. Tripney J, Kwan I, Schucan Bird K (2013) Postabortion family planning counseling and services for women in low-income countries: A systematic review. 
Andrzej Kulczycki

Contraception, 87(1): 17-25.

9. Rominski SD, Lori JR. Review: Abortion care in Ghana: A critical review of the literature. Afr J Reprod Health, 2014; 18(3): 17-35.

10. Millard C, Brhlikova P, Pollock A. Social networks and health policy: The case of misoprostol and the WHO model essential medicine list. Social Science and Medicine, 2015; 132: 190-196.

11. Ipas and Venture Strategies Innovations. Misoprostol use in postabortion care: A service delivery toolkit. Chapel Hill, NC: Ipas, 2011.

12. WHO. 2012. Safe abortion: technical and policy guidance for health system, $2^{\text {nd }}$ ed. Geneva: World Health Organization.

13. Gaye A, Diop A, Shochet T, Winikoff B. Decentralizing

\section{Misoprostol for post-Abortion Care in Africa}

postabortion care in Senegal with misoprostol for incomplete abortion. Int J Gynaecol Obstet, 2014; 126: 223-26.

14. Klingberg-Allvin M, Cleeve A, Atuhairwe S, Tumwesigye NM, Faxelid E, Byamugisha J, Gemzell-Danielsson K. Comparison of treatment of incomplete abortion with misoprostol by physicians and midwives at district level in Uganda: a randomized controlled equivalence trial. Lancet, 2015; 385: 2392-8.

15. Ngwena CG, Brookman-Amissah E, Skuster P (2015)

Human rights advances in women's reproductive health in Africa. International Journal of Gynecology and Obstetrics, 129: 184-187. 